\title{
THE FIFTY-SECOND REGULAR MEETING OF THE SAN FRANCISCO SECTION
}

The fifty-second regular meeting of the San Francisco Section of the Society was held at the University of British Columbia on Saturday, June 18, 1927. The total attendance was thirty, including the following fifteen members of the Society:

Bernstein, Biggerstaff, Blichfeldt, Daniel Buchanan, J. W. Campbell, Carpenter, DeCou, Griffin, McAlister, W. E. Milne, Moritz, Mullemeister, Neikirk, F. S. Nowlan, Winger.

Professor Daniel Buchanan was elected temporary chairman. President Klinck of the University of British Columbia welcomed the visiting members. The Section accepted the cordial invitation of Professor Griffin to hold the next Summer meeting at Reed College. After the meeting the visitors were entertained at a luncheon on the Campus followed by a drive through the city of Vancouver.

Titles and abstracts of papers read at the meeting follow. The papers of Professors Bell, Buchanan, Davis, Dines, Moore, Dr. Trjitzinsky, Mr. Gage, Mr. Jerbert, and Dr. Whyburn were read by title. The papers of Miss Deutsch, Miss Haack, Miss Pennock, Miss Rollins, and Mr. So were sponsored and read by Professor Griffin. Mr. Gage's paper was sponsored by Professor Buchanan.

1. Professor E. T. Bell: Cauchy's cyclotomic function and functional powers.

This paper has appeared in the July-August number of this Bulletin.

2. Professor E. T. Bell: Numerical functions of multipartite integers and compound partitions.

An arithmetic of hypercomplex numbers with $r$ positive integral coefficients is developed. The divisibility properties of functions of such numbers are a logical resultant of those of Dirichlet multiplication and the process of elliptic multiplication and the processes of elliptic functions; they give rise to new aspects of compound partitions. 
3. Professor E. T. Bell: Partition polynomials.

A class of polynomials depending on all the partitions of a given integer into unequal parts is defined. For special values of the $s$ independent variables in the $n$th polynomial, the value is the number of representations of $n$ in the form $c x \cdots x x$ where $c, \cdots$ are integers 0 ; for other special values the $n$th polynomial is the binary quadratic class number for the negative determinant $n$.

\section{Professor Daniel Buchanan: The Trojan asymptotic} satellites.

An earlier paper discussed the asymptotic satellites in the vicinity of the equilateral triangle equilibrium points, but the analysis was not valid for certain small ratios of the finite masses, for example, that of Jupiter and the sun. A different treatment of the problem is now given which is valid for certain small ratios only.

\section{Professor A. F. Carpenter: Complete systems of invariants} and covariants of a certain system of surfaces.

An analytic basis for the projective differential geometry of triads of ruled surfaces whose generators are in one-to-one correspondence has been established in a paper soon to appear in the Transactions. As a sequel the present paper develops with reasonable completeness the theory of the invariants and covariants of such a system of surfaces.

\section{Professor D. R. Davis: Characteristic properties of dif-} ferential equations of the calculus of variations.

Self-adjoint systems of differential equations of the second order are discussed. For a given system of differential equations of the second order the unique existence of an adjoint system is proved. Necessary and sufficient conditions for a given system of the second order to be self-adjoint are established. Characteristic properties of differential equations of the calculus of variations are then derived. It is here shown that if two equations of the form $H\left(x, y, z, y^{\prime}, z^{\prime}, y^{\prime \prime}, z^{\prime \prime}\right)=0, K\left(x, y, z, y^{\prime}, z^{\prime}, y^{\prime \prime}, z^{\prime \prime}\right)=0$ are to be the Euler equations for the integral $I=\int_{x_{1}}^{x_{2}} f\left(x, y, z, y^{\prime}, z^{\prime}\right) d x$, they must have their equations of variation $H_{y} u+H_{z} v+H_{y^{\prime}} u^{\prime}+H_{z} v^{\prime}+H_{y^{\prime \prime}} u^{\prime \prime}$ $+H_{z}^{\prime \prime} v^{\prime \prime}=0, K_{y} u+K_{z} v+K_{y^{\prime}} u^{\prime}+K_{z}{ }^{\prime} v^{\prime}+K_{y}{ }^{\prime \prime} u^{\prime \prime}+K_{z}{ }^{\prime \prime} v^{\prime \prime}=0$, self-adjoint.

7. Professor D. R. Davis: Integrals whose extremals have given differential equations.

That the equations of variation of a given system of differential equations of the second order, $H=K=0$, must be self-adjoint in order that the given equations be the Euler equations for an integral $I$ was established as a necessary condition in the preceding paper. The sufficiency of this property is proved here. Necessary and sufficient conditions for the system $H=K=0$ to be self-adjoint are given. Also the most general form of the 
integrand $f$ for an integral $I$ whose Euler equations are the equations $H=K=0$ is determined.

8. Professor D. R. Davis: A method of determining an integral I whose extremals are a given family of curves.

The problem of finding an integral whose extremals are a given family of curves is here treated. Necessary and sufficient conditions for the two given equations of the form $y^{\prime \prime}=F\left(x, y, z, y^{\prime}, z^{\prime}\right), z^{\prime \prime}=G\left(x, y, z, y^{\prime}, z^{\prime}\right)$ to have solutions $y=y(x), z=z(x)$ which represent the extremals for an integral $I$, of the form expressed in abstract 6 , are derived. Also the possibility of determining linear conbinations of the two given equations $y^{\prime \prime}=F, z^{\prime \prime}=G$, which shall have equations of variation that are self-adjoint, is discussed. As an application of the above theory the following cases are treated: (1) integrals whose extremals are straight lines, (2) integrals whose extremals are semicircles, (3) integrals whose extremals are catenaries.

\section{Miss Helen Deutsch: Note on certain questions of probability.}

This note considers the most probable straight line for a given table of data, and discusses criteria for the line to be unaltered by reversing the selection of the independent variable, also an upper limit for the changes in the constants of the line for given errors in the table. Some curious results of random sampling are also reported.

\section{Professor L. L. Dines: On positive solutions of a system of linear equations.}

This paper presents a simple algorithm for determining whether a given system of linear algebraic equations with real numerical coefficients admits a solution in which each of the unknowns has a positive value, and for determining such a solution when one exists. It will appear in the Annals of Mathematics.

11. Professor L. L. Dines: On completely signed sets of functions.

A set of functions $f_{1}(x), f_{2}(x), \cdots, f_{m}(x)$, continuous on an interval $a \leqq x \leqq b$, is said to be a completely signed set, if there exist $2^{m}$ points $x_{1}$, $x_{2}, \cdots, x_{2^{m}}$, on the interval such that the matrix $\left\|f_{i}\left(x_{1}\right)\right\|$ is a completely signed matrix (as already defined, Annals of Mathematics, vol. 28, p. 42). The author shows that the condition of being completely signed is sufficient (by no means necessary) to ensure the following two properties for the set: (1) every linear combination of the functions changes sign on the interval; (2) there exists a positive continuous function on the interval which is orthogonal to each function of the set. The further remark is made that the notion of a completely signed set may be extended to a set of functions of a general variable. The paper will appear in the Annals of Mathematics. 
12. Mr. W. B. Doggett: On certain caustic surfaces and optical phenomena.

If the farther half of a spherical flask be silvered, and the flask be nearly filled with water, the observer will see at bottom an apparent bubble or globule of immiscible fluid. Experiment indicates that this is the image of the upper surface of the water, distorted by reflection and refraction; and this conclusion is verified mathematically. Preliminary to this study, there is a discussion of caustic surfaces formed by reflection from spherical and parabolic surfaces.

13. Mr. W. H. Gage: Asymptotic satellites near the equilibrium point in the isosceles triangle solution of the problem of three bodies (elliptic case).

If two finite bodies of equal mass move in circles or ellipses about their common center of gravity, then an infinitesimal third body may be initially projected so that it will move in a periodic orbit and remain equidistant from the finite bodies. The paper determines the orbits which are asymptotic to this periodic orbit when the motion of the finite bodies is elliptic.

14. Professor F. L. Griffin: Points of minimum travel for a distributed population.

The General Report of the U. S. Census, after defining the center of population, erroneously states that it is the point at which all the people could be assembled with the minimum aggregate travel, going directly. This suggests the problem of determining points of minimum travel for areas of various shapes and for a population whose density varies in an arbitrary manner. Several cases are discussed.

\section{Miss Bonnie Haack: Certain families of curves having a parabola as their envelope.}

This paper formulates the inverse of the envelope problem for families of coplanar curves, and studies certain families of straight lines, circles, and parabolas having a given parabola as their envelope.

16. Mr. A. R. Jerbert: Projective differential geometry of a system of linear, homogeneous, first-order differential equations.

This paper studies a system of linear, homogeneous, first-order differential equations in three dependent and one independent variables. A fundamental system of independent solutions can be interpreted as the homogeneous coordinates of three points in a plane. This system of equations defines three curves "to within a projectivity." As the independent variable varies, the points describe three associated curves, corresponding points being those given by the same value of the independent variable. The complete system of invariants of the system is obtained and interpreted as expressing projective properties of the associated curves. 
17. Professor R. E. Moritz: The general solution of a certain Diophantine equation in three unknowns.

A certain problem in partitions leads to the equations $x-1=k n$, $y=h n$, where $k, n$, and $h$ are positive integers subject to the condition $(k+1)(n-1)-n^{n-1}(h n+n-1)=0$ but otherwise unrestricted. This paper deals with the general positive integral solutions.

18. Professor R. E. Moritz: Some new formulas for estimating depreciation of physical properties.

As is well known, the reducing balance method of computing depreciation results in an excessive reduction of book values during the early years of an organization by creating what amounts to a secret reserve. This paper shows how this objection may be removed by considering the ratio of depreciation as a function of the time and by determining this function with regard to the nature of the property under consideration.

19. Professor R. E. Moritz: A modification of Glaisher's proof of Sterling's theorem.

Glaisher's so called elementary proof of Sterling's formula for the approximation of large numbers is open to the objection that it assumes without proof the convergence of a certain double series, and assumes as constant a variable which approaches a constant as a limit. The paper offers an elementary proof of the theorem which is free from these objections.

20. Professor L. I. Neikirk: A totally discontinuous function. Preliminary report.

If $f_{1}(x)$ and $f_{2}(x)$ are any two functions of $x$, then the function under discussion will be equal to $f_{1}$ at every point of an interval except at an enumerable set of points which are everywhere dense, where it is equal to $f_{2}$. Between every two points at which it equals $f_{1}$ there is a point at which it equals $f_{2}$, and vice versa. An explicit formula is given for this function.

21. Professor F. S. Nowlan: Arithmetics of rational division algebras of order nine.

In this paper the integral elements (in the Dickson sense) of a certain rational division algebra $D$ are determined. The basal units of $D$ are given by $y^{i} x^{j}(i, j=0,1,2)$, where $y^{3}=\eta \epsilon$ with $\eta$ the product of integral powers of 7 and rational primes of the form $7 m \pm 1$ and $\epsilon$ is the product of rational primes $7 m \pm 2$ and $7 m \pm 3$, with at least one such factor entering to an integral power not divisible by 3 . Further, $x$ satisfies the irreducible cyclic equation $w^{3}+w^{2}-2 w-1=0$. For such value of $\epsilon$ of the form $7 m \pm 1$ there are three sets of integral elements. If, however, $\epsilon$ is of the form $7 m \pm 2$ or $7 m \pm 3$, then for each of its values there is unique set of integral elements, viz., the set $I$ of all elements with a rational integral coordinates, referred, however, to a transformed set of basal units in case $\eta \neq 1$. 
22. Professor F. S. Nowlan: A note on the direct product of a division algebra and a total matric algebra.

This paper establishes certain theorems concerning an algebra expressible as the direct product of a division algebra and a total matric algebra. In particular, it develops a method for finding the primitive idempotent elements of a total matric algebra.

23. Professor F. S. Nowlan: A problem related to matric algebras.

Given the basis of a total matric algebra all similar bases are determined.

\section{Miss Dorothory Pennock: A generalization of the} circular and hyperbolic functions of sectorial areas.

The familiar line definitions are generalized by measuring the sectorial areas from an origin other than the center of the circle or hyperbola. The functions so generalized are studied in detail as to their graphs, periodicity, basic relations, derivatives, integrals, and development in series. A short discussion of similar functions for the parabola is appended.

25. Miss Marguerite Rollins: Functionally conjugate curves. The case of the harmonic mean.

A basic reference curve $B$ and a given curve $G$ are selected, and a conjugate curve $R$ is defined as having its radius vector in any direction the harmonic mean of the corresponding radii vectors of $B$ and $G$. The character of $R$ is studied when $B$ and $G$ are straight lines or circles or certain other conics. When $B$ and $G$ coincide as a single conic, $R$ becomes the polar of the origin.

26. Mr. Hung Shu So: On the projective determination and classification of conics through given points.

This paper presents a detailed discussion of the character of conics as determined by given sets of points, with special attention to degenerate forms, and with simple criteria for the selection of points which will determine any desired type of conic, or vice versa.

27. Dr. W. J. Trjitzinsky: Integrals of non-analytic functions.

Functions non-analytic in the sense of being non-monogenic do not possess a unique derivative at a point. If $C$ be a closed curve, then under certain conditions $\left|\int_{C} F(z) d z\right|<4 \sqrt{2} M \cdot A$, where $M$ is the upper bound of the maximum directional derivative on and within $C$, and $A$ is the area within $C$. This relation is an analoge of Cauchy's theorem and is proved by using essentially Goursat's method in proving Cauchy's theorem. As 
a corollary, we have upper bounds for certain line integrals much closer than the upper bounds given by the ordinary method.

\section{Dr. W. J. Trjitzinsky: Zeros of a function and zeros of its derivatives.}

This paper will appear in full in an early issue of this Bulletin.

29. Dr. W. J. Trjitzinsky: Relations satisfied by coefficients of periodic solutions.

Assuming that there is a periodic solution of a linear homogeneous equation with periodic coefficients containing parameters and differentiating the solution with respect to one of the parameters, the derivative is found to be a periodic solution of a certain linear non-homogeneous differential equation. Solving this by the method of variation of parameters and introducing the condition of periodicity, a relation is found satisfied by the coefficients of a periodic solution containing all of them. This method is, in general, complicated, but the difficulty is avoided by using Fite's recent results concerning periodic solutions. The results of the paper were applied to the Mathieu differential equation of the elliptic cylinder and to Hill's equation of the lunar theory.

\section{Dr. W. J. Trjitzinsky: Representation of functions} determined by their initial values. First paper.

Borel, Denjoy, and de la Vallée Poussin have investigated classes of functions more general than the class of analytic functions; they are characterized by the fact that they are determined by the initial values (of the function and its successive derivatives); de la Vallée Poussin gives a practical method for constructing such functions when the initial values are assigned by means of a series $\alpha_{1} \cos m_{1} x+\alpha_{2} \cos m_{2} x+\cdots$ where $m_{i}$ are integers such that $m_{k+1} / m_{k}>r>1$ for all $k$. In this paper it is shown that the functions under consideration are representable by a series at the form $\alpha_{1} f\left(m_{1} x\right)+\alpha_{2} f\left(m_{2} x\right)+\cdots$ where $|f(x)|,\left|f^{(2 p)}(x)\right|<M$ for all $p$ 's and all real $x$ 's. As a corollary, it is shown that when $m_{i}$ are restricted in a certain way, an analytic function $F(x)$ is expansible on $(-1,+1)$ in a series $F(x)=\sum_{i=1}^{\infty} \alpha_{i} f\left(m_{i} x\right)$, where $\alpha_{i}$ are given by complex integrals. The proof is close to de la Vallée Poussin's proof.

\section{Dr. W. J. Trjitzinsky: Representation of functions de-} termined by their initial values. Second paper.

It is found that when a function $F(x)$ which is determined by its initial values is represented by the series $\sum_{i=1}^{\infty} \alpha_{i} f\left(m_{i} x\right)$, the approximation furnished by taking the first $n$ terms of the series is of the order of $1 / r^{2 n}(r>1)$. Moreover, it is found that the functions $f(x)$ which are admissible in the above representation may be computed in the form $f(x)=\sum_{j=0}^{\infty} C_{j} x^{2 j}$ where 
$C_{j}=(-1)^{j} \sum_{k=1}^{\infty}\left(p_{k}^{2 j} c_{k}\right)$ and $c_{i}, p_{j}$ are any numbers such that $\sum_{i=0}^{\infty}\left|c_{i}\right|$ converges and $\left|p_{i}\right| \leqq 1$; also, it is found that these functions $f(x)$ satisfy on $(-1,+1)$ relations of the form $f(x)=\sum_{i=1}^{\infty} \alpha_{i} f\left(m_{i} x\right)$, where $\alpha_{i}$ are independent of the choice of $f(x)$.

\section{Dr. W. J. Trjitzinsky: Polynomials approximating to} functions analytic or quasi-analytic.

Taking de la Vallée Poussin's representation of functions by means of a series $\sum_{i=1}^{\infty} \alpha_{i} \cos m_{i} x$, let us take the first $n$ terms of the series i.e., a trigonometrical polynomial of order $m_{n}$ which approximates to the function. By Fejer's result it would follow that the maximum of the above approximating polynomial is not greater than $m_{n}$ times its minimum (within a period), and conversely. In this paper it is shown that under certain slight restrictions the above immediate consequence of Fejér's result can be made more precise (though less immediate); namely, a polynomial of order $m_{n}$ approximating to any of the functions of the class under consideration has its maximum not greater than $n$ times its minimum $\left(n<m_{n}\right)$, and conversely.

\section{Dr. G. T. Whyburn: Some theorems on continuous} curves.

In this paper it is shown that if $M$ denotes any plane continuous curve, the following theorems are true. (1) If $G$ denotes the set of all the cut points $[P]$ of $M$ such that $P$ is an irregular point of some sub continuum of $M$, then each point of $G$ belongs to some simple closed curve in $M$, and $G$ is countable. (2) The cut point $P$ of $M$ belongs to no simple closed curve in $M$ if and only if it is an end point of every continuum obtained by adding $P$ to some maximal connected subset of $M-P$. (3) In order that $M$ should fail to separate the plane it is necessary and sufficient that every maximal cyclic curve of $M$ should be a simple closed curve plus its interior. (4) A maximal cyclic curve of $M$ is a simple closed curve if and only if it is the common boundary of two complementary domains of $M$. (5) If $M$ itself is not the boundary of any domain, than in order that every proper subcontinuum of $M$ should be the boundary of a domain it is necessary and sufficient that $M$ should be the sum of three arcs joining two points $A$ and $B$ of $M$ and having no other common points.

34. Dr. G. T. Whyburn: Concerning closed and connected subsets of the cut points of continua.

Let $M$ be a bounded continuum and $K$ any closed and connected subset of the cut points of $M$. The author has shown (Transactions of this Society, vol. 29, 1927, p. 383) that $K$ is an acyclic continuous curve. In this paper it is shown that: (1) $K$ has only a countable number of end points; (2) if $A B$ is any arc of $K$ then $M-A B$ plus some countable subset $T$ of $A B$ is the sum of the elements of a countable upper semi-continuous collection $G^{\prime}$ of mutually exclusive continua each of which contains exactly 
one point of $T$; furthermore, if $G$ denotes the collection obtained by adding to $G^{\prime}$ all those points of $A B-T$, then $M$ itself is a simple continuous arc of elements of $G$ from the element containing $A$ to the one containing $B$;

(3) $M-K$ plus some countable subset $H$ of $K$ is the sum of the elements of a countable upper semi-continuous collection $E^{\prime}$ of mutually exclusive continua each of which contains exactly one point of $H$, and if $E$ denotes the collection obtained by adding to $E^{\prime}$ all those points of $K-H$, then $M$ itself is an acyclic continuous curve of elements of $E$ whose end elements are the elements of $E^{\prime}$ which contain end points of $K$; (4) $M$ is regular at every point of $K-H$.

\section{Professor R. M. Winger: The equianharmonic cubic} and its group.

The equianharmonic cubic admits, as is well known, a monomial group of order 54, which is a subgroup of the Hesse group. A complete system of invariants of this group is obtained and numerous invariant curves are considered. In particular, the pencil of invariant sextics, which contains several interesting members, is discussed and some new properties of the sextic invariant of the Hesse group are found.

\section{Mr. John Biggerstaff : Arithmetic of nonians.}

The writer finds the set of integral elements of the algebra of nonians according to Dickson's set of properties. Application of the theorem that the product of nonions equals the product of the norms of the factors, gives solutions of diophantine problems involving relations of the third degree.

37. Professor J. W. Campbell: On the strength of guy wires. Preliminary report.

A solution is obtained for the restoring couple afforded to a tower by a given pair of symmetric guy wires when the tower leans through a small angle in the plane of the wires.

\section{Professor R. L. Moore: A separation theorem.}

In this paper it will be shown that if $T$ is a totally disconnected closed subset of the boundary of a bounded simply connected domain $D$ and there exists a continuum $K$ containing $T$ and such that $K-T$ is a subset of $D$ then there exists a simple closed curve $J$ containing a subset of $T$ and enclosing a part of $K-T$ and such that $(J+T)-T$ is a subset of $D-(K-T)$.

It is also shown that this proposition does not remain true on the omission of "a part of" before " $K-T$ " even though $T$ consists of a simple point on the outer boundary of $D$. This corrects a statement made on page 473 (see Theorem 3 ) of the author's paper concerning the separation of point sets by curves (Proceedings of the National Academy, vol. 11, 1925). 
39. Dr. G. T. Whyburn: Concerning connected and regular point sets.

This paper will appear in full in an early issue of this Bulletin.

B. A. Bernstein, Secretary of the Section

\section{BEQUESTS TO THE SOCIETY}

At its meeting held on May 6, 1927, the Board of Trustees of the Society adopted the following resolution:

Resolved, that the Treasurer be, and hereby is, instructed to credit to endowment all bequests made to the American Mathematical Society except those specifically designated for other purposes.

Several members have already made provision for bequests to the funds of the Society. The specific action of the Trustees was taken in view of the suggestion of one of these persons that other members wishing to take similar action might know what disposition would be made of unspecified bequests. For such bequests, the following form, which is sufficient in any state, is suggested:

"I give and bequeath to the American Mathematical Society, a corporation incorporated under the laws of the District of Columbia, the sum of ..... dollars."

Still others have written into their wills bequests for specific purposes, such as prizes. Members who desire to make, such bequests are invited to consult the officers of the Society regarding the most urgent needs of mathematics in this country.

W. B. Fite,

Treasurer. 